\title{
giant ovarian
} cystadenoma: report of a case

\begin{abstract}
The ovarian masses are a common finding in the female population being a common reason for consultation in primary care, so it is very important to make an adequate approach to the adnexal masses always trying to catalog them according to their classification can be functional as cysts follicular and pathological such as neoplasms, whether benign, such as ovarian or malignant cystadenomas, such as cystadenocarcinomas. In the present case documentary review, we have presented the clinical case of a 24-year-old woman with a left adnexal mass corresponding to giant cystadenoma $(37 \mathrm{~cm})$, which is defined as an ovarian cystic structure greater than $15 \mathrm{cms}(10)$ which in this particular case generates a compressive effect on the right ureter which leads to a right hydronephrosis, this being one of the most common complications in pelvic masses.
\end{abstract}

Keywords: palabras claves, quiste ovárico, hidronefrosis, cistoadenoma ovarico
Volume 5 Issue 5 - 2018

\author{
Diego Alejandro Saldaña Peñaloza, Leidy \\ Carolina Garcia Mendieta \\ Universidad nacional de Colombia, Colombia
}

\author{
Correspondence: Diego Alejandro Saldaña Peñaloza, \\ Universidad nacional de Colombia, Colombia, \\ Email diegosp2008md@gmail.com
}

Received: June 21, 2018 | Published: September 04, 2018

\section{Introduction}

Ovarian masses are common findings in the female population, remains a common consultation in primary care, which is very important to make a proper approach of adnexal masses always trying to categorize them according to their classification can be functional as follicular cysts and pathological as neoplasms are benign and malignant as ovarian or cystadenomas as cystadenocarcinomas. In the present documental review case of a 24 year old woman with left adnexal mass corresponds to giant cystadenoma $(37 \mathrm{cms})$ which is defined as a cystic structure greater ovarian of $15 \mathrm{cms}(10)$ is present which generates this particular case a compressive effect on the right ureter which leads to a right hydronephrosis.

\section{Case report}

24 year old female patient who attends to overall control, at the time of referred was found asymptomatic in history but I indicated that her mother had died of liver sarcoma no other antecedent family, personal or surgical, referred menarche at 13 years, with regular menstrual cycles without recent changes from cycle frequency or amount of menstrual bleeding, condom use as a method of family planning for 6 years, when no gestational history, clinical examination only increased abdominal girth positive ascetic wave was evident, no changes in abdominal surface vasculature, masses or palpable organ enlargement were evident, no abdominal pain or discoloration of skin or mucosa, thus possible ascites was considered establish and given rise to this initial studies to study origin of ascites were requested. During the next control, no changes compared to previous clinical examination were evident in paraclinical transaminases and bilirubin. the reports in normal parameters, hematology and normal clotting times, quantitative $\mathrm{BHCG}<0.10 \mathrm{mIU} / \mathrm{mL}$ (negative) and abdomen total ultrasound showed limited by the large amount of free fluid in the abdominal cavity, with decreased bilateral renal size and dilated right renal pelvis, study no proper evaluation of abdominal organs was achieved by a free liquid, the above clinical suspicion gave gynecopathologists possible neoplasic whereby extension tests were completed including abdominal tomography contrast to study possible neoplastic lesions. For the next control in clinical examinations showed a normal peripheral blood smear, differential protein and globulin normal blood, a $0.90 \mathrm{mg} / \mathrm{dl}$ for CKD-EPI GFR $88.3 \mathrm{~mL} / \mathrm{min} / 1.73 \mathrm{~m}^{2}$, HIV testing and syphilis serology negative, alpha-fetoprotein $0.9 \mathrm{ng}$ / dl (negative), carcinoembryonic antigen $1.32 \mathrm{ng} / \mathrm{ml}$ (negative), CA $07.1619 .9 \mathrm{IU} / \mathrm{ml}$ (negative), CA $1258.98 \mathrm{UI} / \mathrm{ml}$ (negative) and the $\mathrm{CT}$ abdomen which was evident great mass apparently left adnexal cystic density occupies the whole of the abdominal cavity with mass effect on abdominal organs. Figure $1 \&$ Figure 2 with compressive effect on the right proximal ureter setting moderate hydronephrosis, Figure 3. Therefore requested assessment by the Gynecologic Oncology Service, which program for exploratory laparotomy. Day May 19, 2018 was performed in the patient exploratory laparotomy, where a large cystic mass dependent left annex of about $35 \mathrm{~cm}$ in major diameter was found. The colour pearlescent compromising left ovary, compression and abdominopelvic displacement organs which tumor resection of the left ovary, omentectomy and appendectomy was performed, samples were sent to pathology for initial study by freezing and subsequent histopathology block. The pathology report indicated: cyst benign ovarian serous papillary cystadenoma consistent, residual ovarian parenchyma with simple epithelial follicular cysts and inclusion in the other samples not suggestive evidence for histopathological changes. Neoplasms, acute or chronic conditions; cytology extracted cyst fluid was negative for malignancy. The patient is discharged after two days without, currently present complications of surgical procedure in outpatient clinical follow by the mass of secondary ovarian origin hydronephrosis.

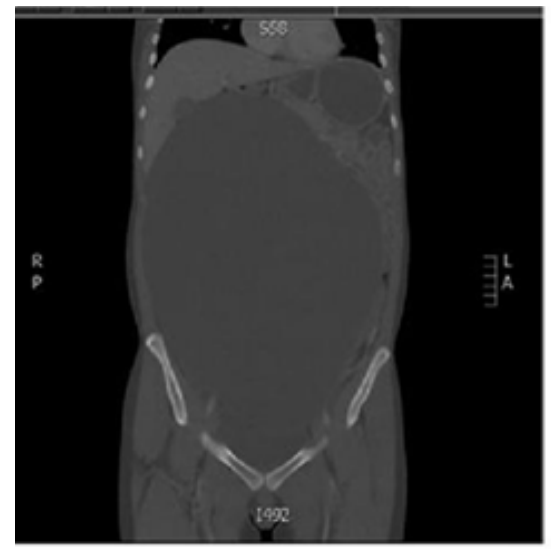

Figure 1 In sagittal section, a cystic mass is observed that occupies a large part of the abdominal cavity, approximate dimensions of $37 \times 13.8 \times 27 \mathrm{~cm}$. In addition, the displacement of intra-abdominal organs. 


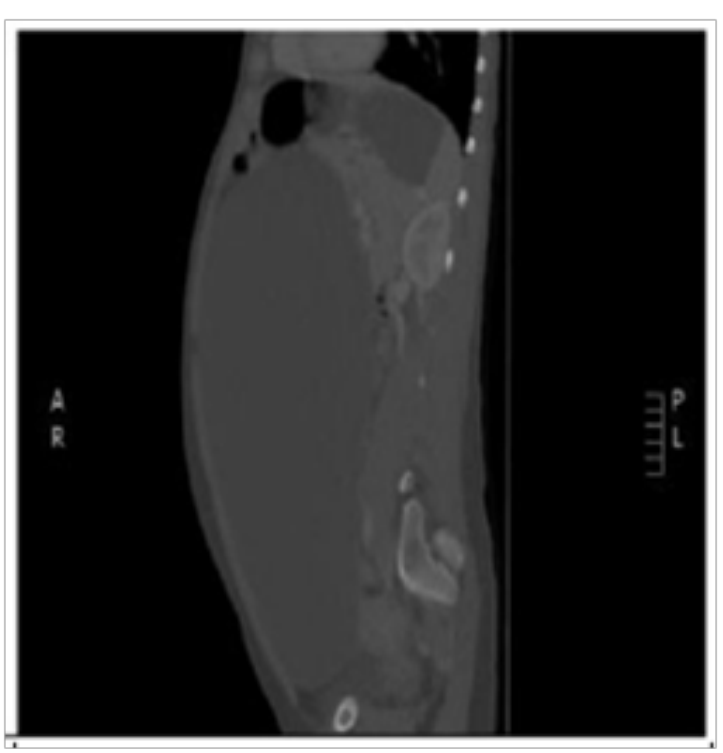

Figure 2 Sagittal section showing evidence of left ovarian dependent mass.

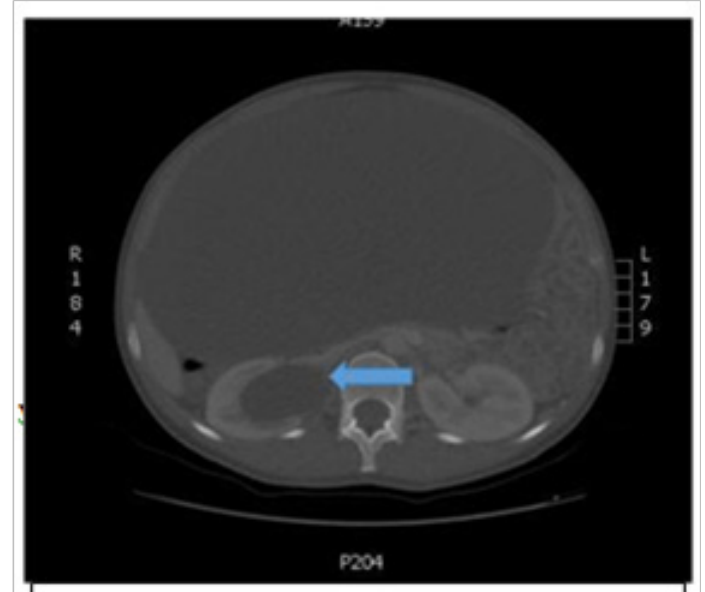

Figure 3 Cross section showing dilatation of the renal pelvis and right ureter.

\section{Discussion}

The patient in whom secondary hydronephrosis was evident to a cystadenoma giant ovarian asymptomatic at the time of medical diagnosis is of great importance in gynecological pathology given its functional implications and commitment in nearby structures given the anatomical relations of the pelvic organs, in this case as evidenced identify anatomic relationships adnexal can explain the origin of hydronephrosis by the patient and his expected improvement post surgical management, currently described in the literature that approximately $15 \%$ of ovarian neoplasms occur before menopause and $58 \%$ occur in less developed countries, with ovarian cancer the most common seventh and eighth in mortality women worldwide according to data from the International Agency for Research on Cancer, ${ }^{1}$ within ovarian neoplasm is most often benign given the great importance of epithelial origin, which one of its greatest representatives he serous cystadenoma. ${ }^{2}$ At the time of study of ovarian masses it is very important to identify their classification, where Ovarian cysts with or without neoplastic origin gain great relevance, which can be functional $(33.2 \%)$ in some cases associated with disruption in the cycle of FSH and LH where an alteration occurs in LH secretion generating increased circulating FSH leading to the growth of the ovarian follicle thereby forming cystic lesions and pathological benign cysts $(19.3 \%)$ or malignant. ${ }^{3,4}$ Benign teratomas on the other hand are lesions germ cell containing the three germ plates (ectoderm, endoderm, mesoderm) and endometriomas are cysts bloody content arising from ectopic endometrium. Also within ovarian masses it is important identify malignant ovarian neoplasms which are not referred to in this discussion as it escapes the purpose of the presentation of the case. ${ }^{3}$

Ovarian cystadenoma within pathology adnexal neoplasia have a frequency of about $24 \%$, which indicated that $70 \%$ can become benign and malignant $6 \%$ as adenocistocarcinomas, the incidence is 15 cases/100.000 and the prevalence is higher in women of reproductive age and who have been treated for infertility with agonists gnRH. ${ }^{3}$ For diagnosis it is regarded as gold standard making pelvic ultrasonography with a sensitivity of $83 \%$ specificity of $89 \%$ (9) however poorly demarcated lesions or echoes inside considered is most relevant findings on computerized tomography (CT) due to its higher specificity for the anatomic description of the lesions. Also with diagnostic imaging decision of tumor markers for study of possible masses of neoplastic origin, it is recommended within these literature indicates you can take the CA125 (low specificity high sensitivity $61-90 \%$ ) and BHCG, but who will be the definitive diagnosis of the lesion histology in patients who are asymptomatic diagnosis is made incidentally as in the case presented. ${ }^{3}$ At the time of clinical examination of patients suspected of gynecological pathology of ovarian origin can be found palpable adnexal masses in bimanual examination 15 to $51 \%$, which should be considered as warning signs of malignancy if besides the abdominal mass HE is abdominal distension, early satiety and ascites, it is always important to consider ovarian masses different differential diagnoses as ovarian cysts functional, neoplastic lesions and lesions no gynecological origin. medical monitoring in cystic lesions of $50 \mathrm{~mm}$ to $70 \mathrm{~mm}$ is suggested, and in which is larger $70 \mathrm{~mm}$ surgical resection of the lesions given the difficulty of ultrasound monitoring as stated in the recommendations of American college of radiology recommend When defining the treatment should take into account the characteristics and size of lesions, being the Medical treatment of choice COCs and larger lesions or suspected neoplasia surgical treatment will be conservative in women of childbearing age, it should also be taken into account when defining surgical handling cysts that have increased size, have increased pain or risk of twisting. is recommended laparoscopy lesions $60-70 \mathrm{~mm}^{5}$ vrs laparotomy as recovery times, less pain and decreased evidence comorbidities, converting laparoscopy to laparotomy is recommended when suspected neoplasia ${ }^{6}$ within the usual complications of adnexal mass is the compression of nearby anatomical structures when they are smaller in size or mass effect displacement of the abdominopelvic organs in larger, in the case reported one right hydronephrosis secondary was evident to large cystic mass in left ovary; within the normal anatomy ureter this comes at the level of the renal pelvis having an abdominal and pelvic downstream region having an anterior relation to the iliac vessels and runs behind the ovary to subsequently cross near the uterine vessels so sagittal reached the base of the broad ligament of the ureter to finally arrive with the neurovascular bundle to the bladder, ${ }^{7}$ hydronephrosis contra lateral, considering that there is increased risk of ovarian cystic lesions hydronephrosis those in which size is $5 \mathrm{~cm}$ 
and still more likely in giant lesions considered which are greater than $15 \mathrm{~cm}^{8}$ which are able to perform a compressive effect displacement intrapelvic or abdominal organs often causing compression on the contra lateral ureter to the location of the lesion which is evidenced in the case presented, in the moment recognizes that full or partial obstruction of the ureter generate urinary flow reduction being caused hydronephrosis, urolithiasis and chronic infections that can lead to progressive deterioration of renal function by progressive increase pyelocalyceal pressure. ${ }^{8}$ From the pathological point of view it is known that the urinary obstruction may be secondary to intrinsic or extrinsic disorders, diseases retroperitoneal tumors nearby anatomical structures, pregnancy among others, which in many cases leads to dilation of being inside the latter renal pelvis by the difficulty of leakage of urine, which increases the pressure level of the renal pelvis carrying a backward return of urine into the collecting ducts which finally leads to atrophy and renal interstitial fibrosis in severe cases, all secondary to the interaction between the cellular response to a noxious stimulus, ${ }^{9,10}$ which explains the decreased renal ultrasonography demonstrated in size taken to the patient, waiting box hydronephrosis improvement after surgical resection of serous cystadenoma not given condition of renal function which is denoted in the value of creatinine together with the glomerular filtration rate exiting this annotated in the presentation of the case and the reversibility of the associated vascular defects to kidney damage in the urinary obstructive pathology to remove the cause of the obstruction, ${ }^{11}$ whereby is expected to follow up the case reported improvement not given the presence of associated comorbidities, age of the patient and no other apparent explanation of the origin of different pelvic mass hydronephrosis apparent.

\section{Conclusion}

Ovarian masses are a common reason for consultation in primary care, which must be identified for their respective management. In addition to recognize its complications commitment to nearby anatomical structures to be the fundamental morphological knowledge to identify potential target organs to masses of ovarian origin, In this particular case of a cystadenoma giant ovary given its large ultimately led to compression of the contra lateral ureter presented himself as a moderate hydronephrosis in a completely asymptomatic patient, which was presented the clinical case which was made size a discussion about hydronephrosis as a complication of ovarian lesions given relations anatomy of organs abdominopelvic.

\section{Acknowledgements}

None.

\section{Conflict of interest}

The author declares that there is no conflict of interest.

\section{References}

1. Perez G, Sierra J, Perez M. Carcinogénesis serous ovarian tumors: surgical implications, recent developments and future challenges for diagnosis and treatment. Mexico Obstetrics and Gynecology. 2018;86(6):389-400.

2. Swamy G, Satyyanarayana N. Clinicopathological analysis of ovarian tumors - A study on five years samples. Nepal Med Coll J. 2010;12(4):221-223.

3. Shannon M. Grabosch Medscape. Chief Editor: Nicole W Karjane. 2017.

4. Hassan S, Abduljabbar. Review of 244 cases of ovarian cysts. Saudi Med J. 2015;36(7):834-838.

5. Guy Rofe, Ron Auslender, Martha Dirnfeld. Benign ovarian cysts in reproductive-age women Undergoing Assisted Reproductive Technology treatment. Open Journal of Obstetrics and Gynecology. 2013;3:17-22.

6. The American College of Obstetricians and Gynecologists evaluation and management of adnexal masses. Practice Bulletin No. 174: Evaluation and Management of Adnexal Masses. Obstet Gynecol. 2016;128(5):e210-e226.

7. Fröber R. Surgical anatomy of the ureter. BJU International. 2007;100(4):949-965.

8. Hayeonkim, Moon Kyoung Cho, Eun Bae Hui, et al. Caused by a giant hydronephrosis ovarian cyst. International Braz J Urol. 2016;42(2).

9. Alpers C, Chang A, Kidney Kumar V. Structural and functional pathology Barcelona. Spain: Elsevier. 2015;39(5):355-361.

10. Klahr S. Obstructive nephropathy. Intern Med. 2000;39(5):355-361.

11. Krajewski W, Wojciechowska J, Dembowski J, et al. Hydronephrosis in the course of ureteropelvic junction obstruction: An underestimated problem? Current opinions on the pathogenesis, diagnosis and treatment. Adv Exp Med Clin. 2017;26(5):857-864. 